\title{
Nonlinear Circuit Analysis via Perturbation Methods and Hardware Prototyping
}

\author{
K. Odame ${ }^{1}$ and P. E. Hasler ${ }^{2}$ \\ ${ }^{1}$ Thayer School of Engineering, Dartmouth College, Hanover, 03755, USA \\ ${ }^{2}$ School of Electrical and Computer Engineering, Georgia Institute of Technology, Atlanta, 30332-0250, USA
}

Correspondence should be addressed to K. Odame, odame@dartmouth.edu

Received 3 June 2009; Accepted 8 December 2009

Academic Editor: Benjamin J. Blalock

Copyright (C) 2010 K. Odame and P. E. Hasler. This is an open access article distributed under the Creative Commons Attribution License, which permits unrestricted use, distribution, and reproduction in any medium, provided the original work is properly cited.

\begin{abstract}
Nonlinear signal processing is necessary in many emerging applications where form factor and power are at a premium. In order to make such complex computation feasible under these constraints, it is necessary to implement the signal processors as analog circuits. Since analog circuit design is largely based on a linear systems perspective, new tools are being introduced to circuit designers that allow them to understand and exploit circuit nonlinearity for useful processing. This paper discusses two such tools, which represent nonlinear circuit behavior in a graphical way, making it easy to develop a qualitative appreciation for the circuits under study.
\end{abstract}

\section{Introduction}

Portable and implantable, always-on electronics stand to benefit from analog signal processing, when only low levels of precision are necessary $[1,2]$. To achieve sophisticated signal processing with low power and area overhead, an analog processor can exploit the fundamental nonlinear dynamics that are found in devices and simple circuits. So, the circuit designer must depart from the traditional linear systems paradigm, and learn to analyze and understand circuits from a nonlinear dynamical systems theory perspective.

In order to make nonlinear circuit design relevant to an engineer, it must be taught intuitively enough to foster creativity, yet rigorously enough to be of practical benefit. The two most popular tools for studying nonlinear circuits are harmonic balance and Volterra series. Within certain limitations, they are rigorous, but not necessarily intuitive. Since harmonic balance is simulation-based, it can be used to predict nonlinear behavior without ever requiring a deep understanding [3] of the circuit. Volterra series is an analytical tool that quickly leads to high entropy [4] mathematical expressions, from which the causative physical phenomena are hard to discern, much less purposefully manipulate.
To bridge the gap between rigor and intuition, we can use visual representation techniques. If the appropriate visualization is formed from rigorous definitions of a circuit's dynamics, then the human vision system, with its pattern recognition ability, will perceive the circuit's qualitative behavior [5].

We will present a filter block diagram for analyzing harmonic distortion that is derived from perturbation analysis. Unlike Volterra series kernels, our filter block diagram does not include multidimensional Fourier transforms, and so is accessible to an introductory-level engineering audience. In the second part of this paper, we will discuss the creation and use of phase plane plots of nonlinear circuits. We will describe how to rapidly create the phase plane plots with a reconfigurable hardware platform, instead of with a numerical simulator.

This paper is an expansion of the work presented in [6].

\section{Regular Perturbation}

Whenever designers want to get an analytical handle on the sources and causes of distortion, the most commonly-used tool is Volterra series analysis. If a problem is tractable using 


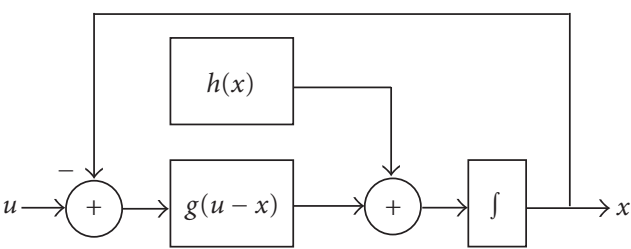

Figure 1: General block diagram form of a first-order circuit. The primary processing block is $g(\cdot)$, which is a nonlinear function of the input $u$ and of $x$ via feedback. The nonlinearity $h(x)$ models such nonidealities as finite output impedance.

Volterra series, then it can also be solved with perturbation theory, which will yield asymptotically-identical results [7].

There are certain problems for which Volterra series are ill-suited-multiple-time-scale behavior and multiple steady states, for instance [8] — that can be solved with perturbation theory. Despite the power of perturbation theory, it is still a relatively obscure concept in discussions about nonlinearity and distortion in analog circuits.

We therefore find it worthwhile to give a basic treatment of regular perturbation-the simplest perturbation method-as applied to distortion analysis of first-order analog circuits. In addition, a filter block diagram representation of the circuit will naturally evolve from our analysis, making it visually clear how the distortion terms are manifested, and how well-known tenets of low-distortion design, such as feedback, come about.

Consider the initial value problem

$$
\dot{x}=f(t, x, \epsilon) ; \quad x\left(t_{0}\right)=x_{0}(\epsilon),
$$

where $\epsilon$ is a small perturbation parameter such that $\epsilon=0$ yields an analytically-soluble equation. If $f$ is sufficiently smooth (the specific smoothness requirements of $f$ are discussed in [9]), then the problem has a unique solution $x(t, \epsilon)$. As the solution for $\epsilon \neq 0$ may not be analytical, it can be approximated as a power series in $\epsilon$ to an accuracy of $\mathrm{O}\left(\epsilon^{n+1}\right)$. That is, we can write the solution as

$$
x(t, \epsilon)=\underbrace{\sum_{i=0}^{n}\left(x_{i}(t) \epsilon^{i}\right)}_{\hat{x}(t, \epsilon)}+\mathrm{O}\left(\epsilon^{n+1}\right),
$$

where $\hat{x}(t, \epsilon)$ is the approximate solution. To conduct regular perturbation, we apply the substitution $x(t, \epsilon) \approx \hat{x}(t, \epsilon)$ to (1). The resulting system is then solved by equating like powers of $\epsilon$. The following sections will illuminate this idea.

\section{The Basic First-Order Circuit}

Most common first-order analog ciruits (simple amplifiers, buffers, switches, etc.) are of the form depicted in Figure 1. The governing equation is

$$
\dot{x}=g(u-x)+h(x),
$$

where $u$ is the a.c. input signal, $x$ is the a.c. output signal and $g(\cdot)$ and $h(\cdot)$ are nonlinear functions. The dependence of the system on the output, other than through feedback to the input, is modeled by $h(x)$. In practice, $h(x)$ is typically some nonideality such as finite output resistance.

In order to apply perturbation analysis to (3), we begin by assuming that the input signal has a small amplitude. This is expressed as $u=\epsilon v$, where $\epsilon$ is a small perturbation parameter and $v$ is a suitably-scaled version of the input signal. Note that with the definition of $u,(3)$ is solvable via separation of variables for the special case $\epsilon=0$.

With the introduction of the perturbation parameter $\epsilon$, we can approximate the solution to (3) with the power series

$$
x(t) \approx \sum_{i=1}^{n} \epsilon^{i} x_{i}(t) .
$$

Note the $\epsilon^{0}$ term of (4) is set to 0 . This corresponds to analyzing a circuit about its d.c. bias point, where the d.c. bias point is shifted to the origin. For ease of notation, define $z=u-x$. The approximation of $z$ is defined similarly to (4), with $z_{1}=v-x_{1}$ and $z_{i}=-x_{i}$, for all $i>1$.

If $\epsilon$ is sufficiently small, then the functions $g(z)$ and $h(x)$ can be approximated by their truncated Taylor series as

$$
\begin{aligned}
& g(z) \approx g_{1} z+g_{n-1} z^{n-1}+g_{n} z^{n}, \\
& h(x) \approx h_{1} x+h_{n-1} x^{n-1}+h_{n} x^{n} .
\end{aligned}
$$

Functions $g$ and $h$ are assumed to be dominantly $(n-1)$ thorder nonlinearities, with $g_{i}=g^{(i)}(0) / i$ ! and $h_{i}=h^{(i)}(0) / i$ !. Equation (5) assumes $g(0)=h(0)=0$, which, again, corresponds to analyzing a circuit about its d.c. bias point.

Substituting (4) and (5) into (3) and collecting powers of $\epsilon$, we get the following set of first-order linear equations

$$
\begin{aligned}
\dot{x}_{1}+\left(g_{1}-h_{1}\right) x_{1}= & g_{1} v, \\
\vdots & \\
\dot{x}_{k}+\left(g_{1}-h_{1}\right) x_{k}= & 0 \quad \forall k<n-1 \\
\vdots & \\
\dot{x}_{n-1}+\left(g_{1}-h_{1}\right) x_{n-1}= & g_{n-1} z_{1}^{n-1}+h_{n-1} x_{1}^{n-1} \\
\dot{x}_{n}+\left(g_{1}-h_{1}\right) x_{n}= & g_{n} z_{1}^{n}-n g_{n-1} z_{1}^{n-1} x_{2} \\
& +h_{n} x_{1}^{n}-n h_{n-1} x_{1}^{n-1} x_{2} .
\end{aligned}
$$

The $\epsilon^{1}$ equation is the linearized portion of (3) with input $v$. Taking the Laplace transform of this equation, we write

$$
X_{1}(s)=g_{1} H(s) V(s),
$$

where $H(s)=1 /\left(s+g_{1}-h_{1}\right)$.

The $\epsilon^{k}$ equations $(k<(n-1))$ are filters with 0 input. As such, the steady state solutions of these equations is 0 .

\section{Harmonic Distortion Terms}

The inputs of the $\epsilon^{n-1}$ equation are terms of $z_{1}^{n-1}$ and $x_{1}^{n-1}$. To understand the implications of these terms to harmonic 


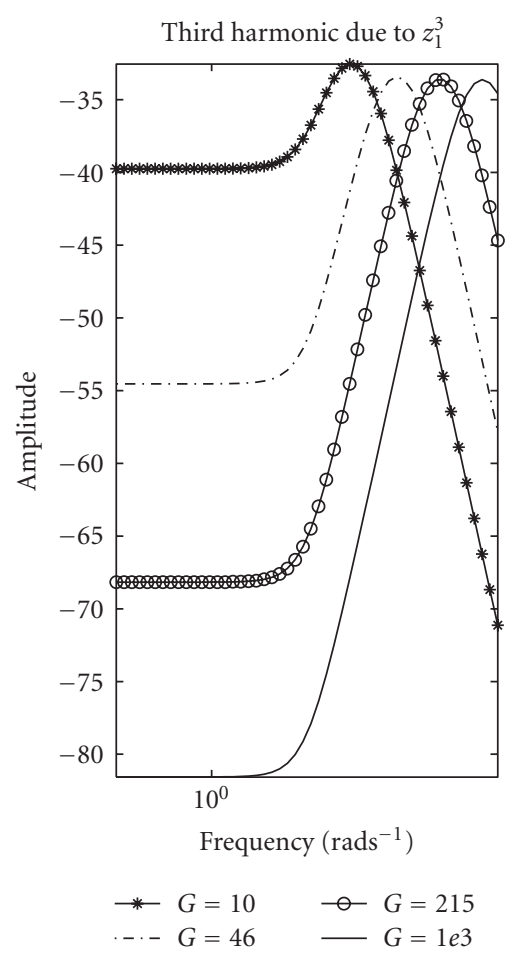

(a)

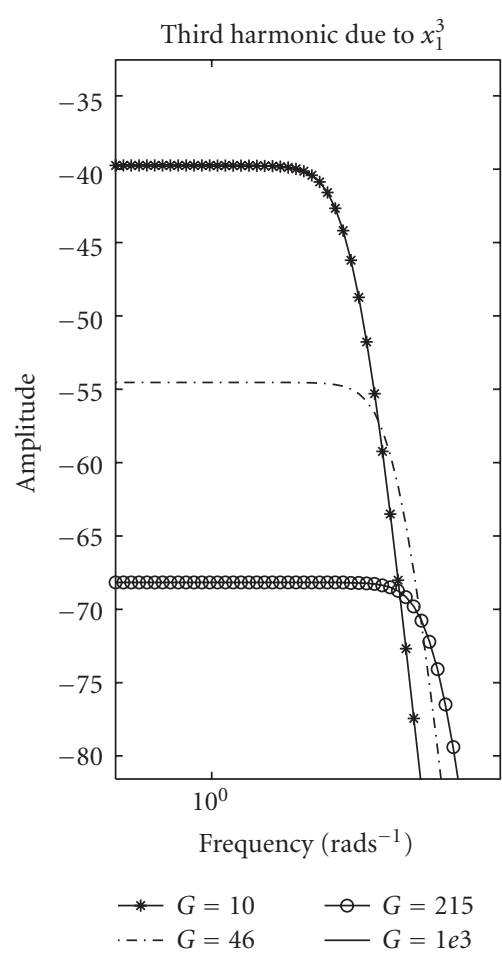

(b)

Figure 2: Magnitude-frequency plots of the third harmonic. The "gain", $G$ of the $g(z)$ function is varied from 10 to 1000 . This causes the band-pass shape of the $z_{1}^{3}$-contributed harmonic to shift to the right, while that contributed by $x_{1}^{3}$ falls in magnitude.

distortion, assume a single-tone input, $v=\cos (\omega t)$. This elicits the signals

$$
\begin{aligned}
x_{1} & =g_{1}|H(j \omega)| \cos (\omega t+\phi(j \omega)), \\
z_{1} & =\left|1-g_{1} H(j \omega)\right| \cos \left(\omega t+\phi_{z 1}(j w)\right), \\
& =|\underbrace{\left(s-h_{1}\right) H(j \omega)}_{H_{z 1}(j \omega)}| \cos \left(\omega t+\phi_{z 1}(j w)\right),
\end{aligned}
$$

Here we have defined $H_{z 1}(s)=\left(1-g_{1} H(s)\right)$. The phases $\phi(s)$ and $\phi_{z 1}(s)$ are the arguments of $H(s)$ and $H_{z 1}(s)$, respectively. The signals $x_{1}$ and $z_{1}$ are single tones of frequency $\omega$ as well, since they are merely linearly-filtered versions of $v$.

Raising $z_{1}$ and $x_{1}$ each to the $(n-1)$ th power produces harmonics as follows. If $(n-1)$ is odd(even), then odd(even) harmonics up to the $(n-1)$ th harmonic are generated. The amplitude of the $m \omega$ frequency term in $x_{1}^{n-1}$ is

$$
\frac{(n-1) ! g_{1}}{((n+m-1) / 2) !((n-m-1) / 2) ! 2^{n-2}}|H(j \omega)|,
$$

while that of the $m \omega$ frequency term in $z_{1}^{n-1}$ is

$$
\frac{(n-1) !}{((n+m-1) / 2) !((n-m-1) / 2) ! 2^{n-2}}\left|H_{z 1}(j \omega)\right| .
$$

After filtering in the $\epsilon^{n-1}$ equation, the amplitudes of these terms will be, respectively,

$$
\frac{(n-1) ! h_{n-1} g_{1}}{((n+m-1) / 2) !((n-m-1) / 2) ! 2^{n-2}}|H(j \omega)||H(j m \omega)|,
$$

$$
\frac{(n-1) ! g_{n-1}}{((n+m-1) / 2) !((n-m-1) / 2) ! 2^{n-2}}\left|H_{z 1}(j \omega)\right||H(j m \omega)| \text {. }
$$

Analogous to that of the $\epsilon^{n-1}$ equation, the input to the $\epsilon^{n}$ equation has terms in $z_{1}^{n}$ and $x_{1}^{n}$. In general, the $x_{2}$ terms are identically zero, except for the special case $n=3$.

\section{Feedback and Distortion}

We now make some observations about the harmonic distortion results that were discussed in the previous section.

In the $\epsilon^{n-1}$ equation, the amplitude of the $m$ th harmonic that the $z_{1}^{n-1}$ term contributes is given by (12). We plot this amplitude expression, along with that of (11), as a function of frequency in Figure 2 for the third-order harmonic generated by a dominantly-third order nonlinearity. That is, $n=4$ and $m=3$. Also, we chose $h_{1}=1, h_{3}=1 / 3, g_{1}=G$, $g_{3}=G / 3$, where $G$ was varied from 10 to 1000 .

Notice from the figure that if $g_{1} \gg h_{1}$, then, for a given frequency, the amplitude of the $z_{1}^{n-1}$-contributed harmonic is greatly reduced. In fact, if we ensure $g_{i} \gg h_{i}$ for all $i$, then the 


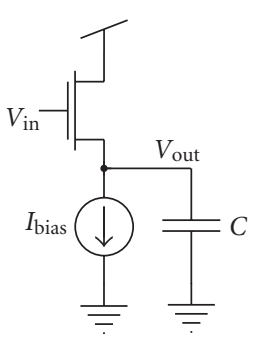

(a)

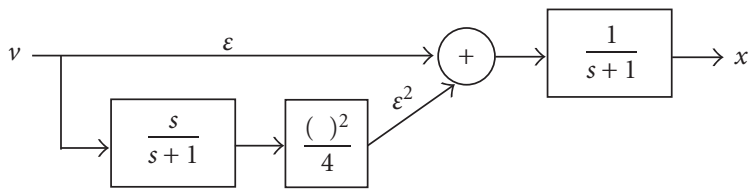

(b)

FIGURE 3: Source follower amplifier. (a) Circuit schematic. (b) Block diagram representation of source follower output. The fundamental harmonic is a low-pass filtered version of the input. The second order terms are generated by high-pass filtering the input, squaring and then low pass filtering. The total output is a power series of $\epsilon$ terms.

harmonic contribution of the $x_{1}^{n-1}$ terms is negligible. This would mean that the distortion is effectively due only to $z_{1}$, whose associated harmonics are band-pass filtered. This in turn means that the distortion can be kept small if the circuit is operated well below the corner frequency.

These two notions-that frequency and feedback gain can be sacrificed for higher linearity-conform with the traditional rules-of-thumb for low-distortion design.

\section{Illustrative Examples}

6.1. Source Follower Amplifier. According to KCL, the circuit equation of the source follower amplifier in Figure 3(a) is

$$
C \frac{d V_{\text {out }}(t)}{d t}=F\left(V_{\text {in }}, V_{\text {out }}\right)-I_{\text {bias }},
$$

where the function $F$ is defined as

$$
F\left(V_{\text {in }}, V_{\text {out }}\right)=\frac{K}{2}\left(\kappa V_{\text {in }}(t)-V_{\text {out }}(t)-V_{\text {th }}\right)^{2},
$$

if $M_{1}$ is in above-threshold saturation, and

$$
F\left(V_{\text {in }}, V_{\text {out }}\right)=I_{\mathrm{o}} e^{\left(\kappa V_{\text {in }}(t)-V_{\text {out }}(t)\right) / U_{\mathrm{T}}},
$$

if it is in subthreshold saturation. The parameter $K$ depends on transistor dimensions and doping and $V_{\text {th }}$ is the threshold voltage. Also, $\kappa, I_{0}$, and $U_{\mathrm{T}}$ have their usual meanings from the EKV MOSFET model [10].

Note that $I_{\text {bias }}=F\left(V_{\mathrm{g}}, V_{\mathrm{S}}\right)$, where $V_{\mathrm{g}}$ and $V_{\mathrm{S}}$ are the d.c. bias-points of the gate and source of $M_{1}$, respectively. Let us define a characteristic voltage, $V_{\mathrm{c}}$, as

$$
V_{\mathrm{c}}= \begin{cases}\frac{\left(\kappa V_{\mathrm{g}}-V_{\mathrm{S}}-V_{\mathrm{th}}\right)}{2}, & \text { above threshold } \\ U_{\mathrm{T}}, & \text { subthreshold. }\end{cases}
$$

Now, (13) can be nondimensionalized [9] by making the substitutions

$$
\tau=\frac{I_{\text {bias }}}{\left(C V_{\mathrm{c}}\right)} \cdot t ; \quad u=\frac{\kappa \nu_{\text {in }}}{V_{\mathrm{c}}} ; \quad x=\frac{\nu_{\text {out }}}{V_{\mathrm{c}}},
$$

where $v_{\text {in }}$ and $v_{\text {out }}$ are the a.c. portions of $V_{\text {in }}$ and $V_{\text {out }}$. This gives the state-space equation of the source follower as

$$
\frac{d x}{d \tau}=u-x+\frac{(u-x)^{2}}{4},
$$

for above threshold, and

$$
\frac{d x}{d \tau}=u-x+\frac{(u-x)^{2}}{2},
$$

for the truncated Taylor expansion in subthreshold. The point is that, regardless of region of operation of $M_{1}$, the nonlinear equation that describes the source follower has the same functional form. Relating the source follower equations to (3), we have $g(z) \sim z+z^{2}$ and $h(x)=0$. As such, we expect the harmonic distortion terms to have a band-passlike dependence on frequency. To show this, we will apply regular perturbation to (18).

First, define $u=\epsilon v$, where the small parameter $\epsilon$ is a scaled version of the input amplitude. That is, $\epsilon=A_{\text {in }} / V_{\mathrm{c}}$. Also, taking $x=\epsilon x_{1}+\epsilon^{2} x_{2}$ and $z=u-x$ and equating like powers of $\epsilon$ up to $\epsilon^{2}$, we have

$$
\begin{gathered}
\epsilon^{1}: \dot{x}_{1}=v-x_{1}, \\
\epsilon^{2}: \dot{x}_{2}=\frac{z_{1}^{2}}{4}-x_{2},
\end{gathered}
$$

as depicted in Figure 3(b). Assume a pure-tone input, $v=$ $\cos (\omega t)$. Equation (20) is the linear portion of the amplifier. Equation (21) is a linear filter with input $z_{1}^{2} / 4$. The squaring produces a second-harmonic term as well as a d.c. offset. In addition, since $z_{1}=v-x_{1}$, the second harmonic generated by the squaring is high-pass filtered. The overall effect is that $x_{2}$ is a band-pass filtered version of a second harmonic of $v$. Figure 4 is a plot of experimental data that corroborates our analysis. There is error in the second harmonic measurement due to the small amplitudes involved.

6.2. Unity Gain Buffer. Consider the unity-gain buffer depicted in Figure 5(a). It is formed by placing an operational transconductance amplifier (OTA) in negative feedback. If we operate the OTA above threshold, the describing equation is

$$
C \frac{d V_{\text {out }}}{d t}=\sqrt{\kappa \beta I_{\text {bias }}}\left(V_{\text {in }}-V_{\text {out }}\right) \sqrt{1-\frac{\kappa \beta\left(V_{\text {in }}-V_{\text {out }}\right)^{2}}{4 I_{\text {bias }}}},
$$

while it is

$$
C \frac{d V_{\text {out }}}{d t}=I_{\text {bias }} \tanh \left(\frac{\kappa\left(V_{\text {in }}-V_{\text {out }}\right)}{2 U_{\mathrm{T}}}\right),
$$

for subthreshold operation. Notice that we have ignored the output conductance term, which is considered very small for OTAs. 


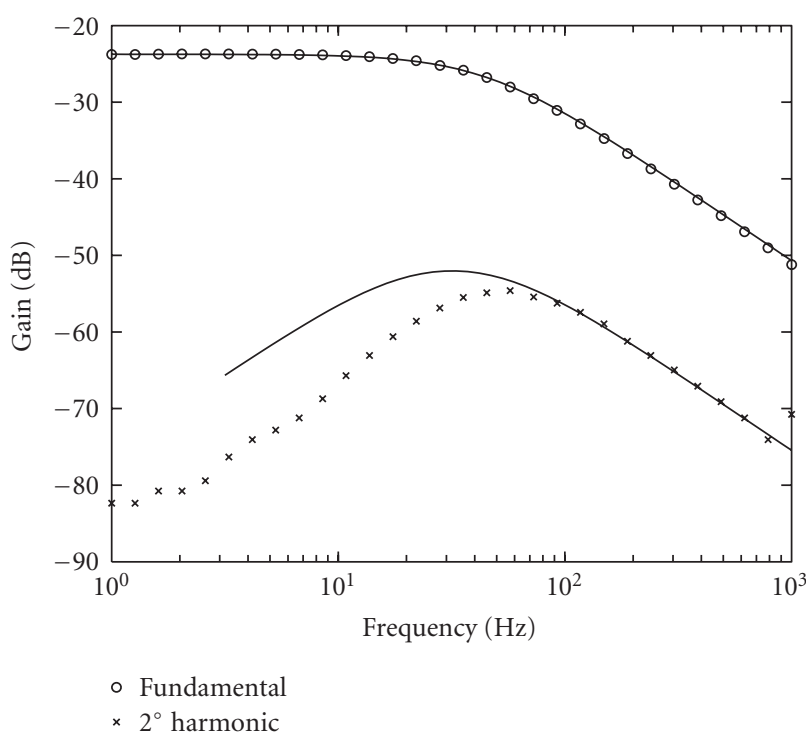

FIGURE 4: Magnitude-frequency response of source follower. Analytical prediction is in bold, and experimental data is plotted as " $x$ "s and " $o$ "s. The fundamental harmonic is a low-pass filtered version of the input. The second harmonic has a bandpass shape, as predicted by perturbation analysis.

We can define a characteristic voltage, $V_{c}$, as

$$
V_{\mathrm{c}}= \begin{cases}\frac{2 U_{\mathrm{T}}}{\kappa}, & \text { subthreshold } \\ \sqrt{\frac{I_{\text {bias }}}{\kappa \beta}}, & \text { above threshold. }\end{cases}
$$

Then, with the following definitions

$$
\tau=\frac{I_{\text {bias }}}{\left(C V_{\mathrm{c}}\right)} \cdot t ; \quad u=\frac{\nu_{\text {in }}}{V_{\mathrm{c}}} ; \quad x=\frac{\nu_{\text {out }}}{V_{\mathrm{c}}},
$$

the nondimensional form of the unity-gain buffer's describing equations (taken to the first few Taylor series terms) is

$$
\frac{d x}{d \tau}= \begin{cases}(u-x)-\frac{(u-x)^{3}}{4}, & \text { above threshold } \\ (u-x)-\frac{(u-x)^{3}}{3}, & \text { subthreshold. }\end{cases}
$$

Again, the functional form of the equations is identical, regardless of region of operation.

To calculate distortion terms, assume $u=\epsilon v$ is a puretone signal and proceed as usual. For subthreshold, the separated equations of $\epsilon$ are

$$
\begin{gathered}
\epsilon^{1}: \dot{x}_{1}=v-x_{1}, \\
\epsilon^{2}: \dot{x}_{2}=0-x_{2}, \\
\epsilon^{3}: \dot{x}_{3}=\frac{z_{1}^{3}}{3}-x_{3} .
\end{gathered}
$$

These equations are depicted in the block diagram of Figure 5(b). Equation (27) is the linear portion of the

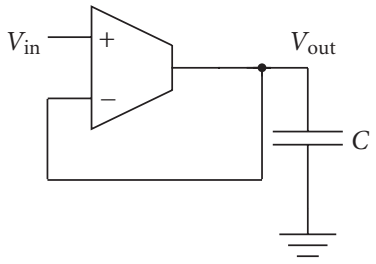

(a)

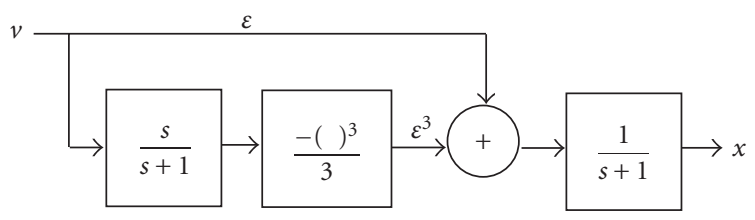

(b)

FIGURE 5: Unity gain buffer. (a) Circuit schematic. (b) Block diagram representation of output. The fundamental harmonic is a low-pass filtered version of the input. The third-order terms are generated by high-pass filtering the input, cubing and then low pass filtering. The total output is a power series of $\epsilon$ terms.

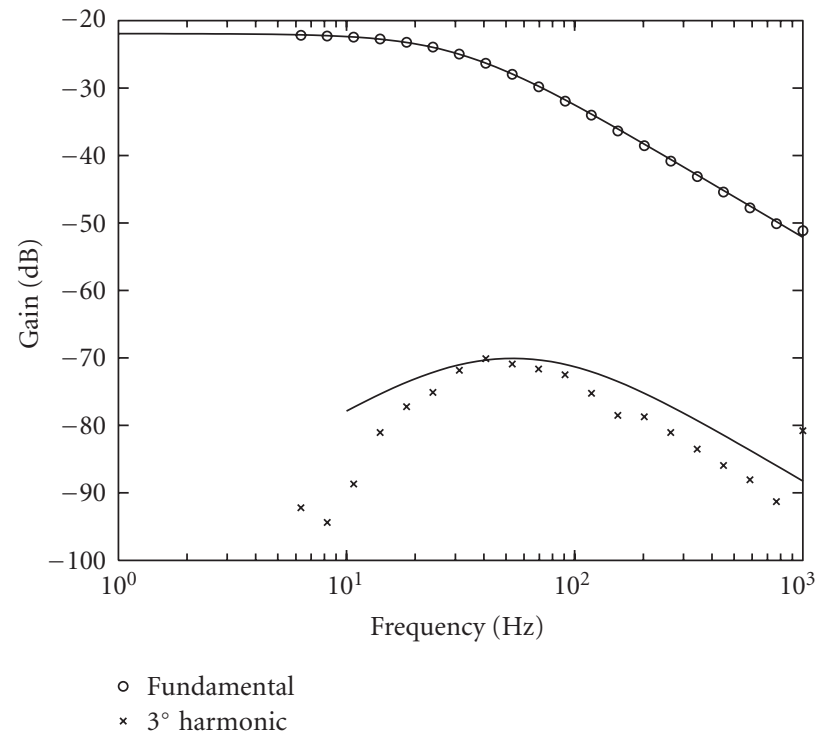

Figure 6: Magnitude-frequency response of unity-gain buffer. Analytical prediction is in bold, and experimental data is plotted as " $x$ "s and "o"s. The fundamental harmonic is a low-pass filtered version of the input. The third harmonic has a bandpass shape, as predicted by perturbation analysis.

amplifier. Equation (28) is a linear filter with 0 input; it contributes no harmonics at steady state. Equation (29) is a linear filter with input $z_{1}^{3} / 3$. The cubing produces a thirdharmonic term as well as a fundamental-frequency term (this fundamental-frequency term will cause gain compression, which is not discussed in this paper). Since $z_{1}=v-x_{1}$, the overall effect is that $x_{3}$ is a band-pass filtered version of a third harmonic of $v$, as shown in Figure 6. 


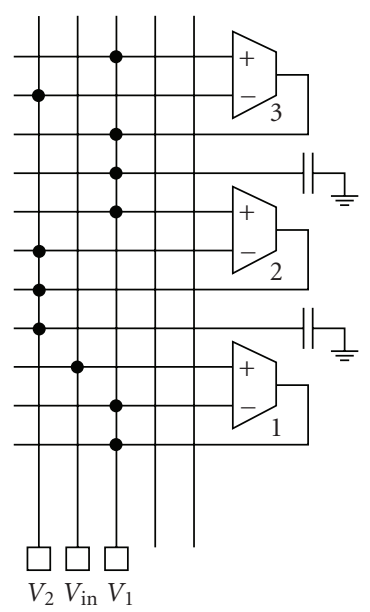

Figure 7: Compiled Circuit on an FPAA. Component terminals can be connected or disconnected using the switch matrix. In this illustration, connections are indicated by solder dots. The gain of each amplifier is programable over a continuous range of values over a few decades.

6.3. Note on Above-Threshold versus Subthreshold Operation. The harmonic behavior of a circuit is similar for aboveand subthreshold operation. In absolute numbers, however, above threshold operation yields less distortion. This is because the parameter $\epsilon=A_{\text {in }} / V_{\mathrm{c}}$ is much smaller for above threshold than for subthreshold. Since the harmonics are multiplied by $\epsilon^{i}$, the smaller $\epsilon$ seen in above threshold operation translates to lower distortion.

\section{Field Programmable Analog Array}

We have developed a field programmable analog array (FPAA) that can be configured to synthesis and analyze a vast variety of circuits [11]. Figure 7 depicts a simple, second-order filter compiled on our FPAA. More complex circuit configurations are possible, and would involve a larger number of the over 400 components in the FPAA. In this part of the paper we demonstrate the utility and versatility of the FPAA in analyzing the dynamics of a number of fundamental circuit blocks.

\section{One-Dimensional Systems}

8.1. Simple Current Mirror. Consider the simple current mirror depicted in Figure 8(a). From Kirchhoff's Current Law (KCL), it obeys the following differential equation:

$$
C \frac{d V_{\mathrm{g}}}{d t}=I_{\mathrm{b}}-f\left(V_{\mathrm{g}}\right),
$$

where $f\left(V_{\mathrm{g}}\right)$ is the drain current of transistor M1. Assuming M1 and M2 are identical and are both saturated, we have $f\left(V_{\mathrm{g}}\right)=I_{\text {out }}$, which gives

$$
C \frac{d V_{\mathrm{g}}}{d t}=I_{\mathrm{b}}-I_{\mathrm{out}}
$$

For subthreshold operation in saturation, the current through transistors M1 and M2 is [10]

$$
f\left(V_{\mathrm{g}}\right)=I_{\text {out }}=I_{\mathrm{o}} e^{\left(\kappa V_{\mathrm{g}}-V_{\mathrm{S}}\right) / U_{\mathrm{T}}},
$$

where $I_{0}$ is a pre-exponential constant dependent on the transistor's size and on doping concentrations. Also, $\kappa$ is the body-effect coefficient and $U_{\mathrm{T}}$ is the thermal voltage. $V_{\mathrm{S}}$ is the source voltage, which, for this case, is zero. Setting $V_{\mathrm{S}}=0$ and taking the derivative of (32) with respect to time, we get

$$
\begin{aligned}
\frac{d I_{\text {out }}}{d t} & =\frac{\partial}{\partial V_{\mathrm{g}}}\left(I_{\mathrm{o}} e^{\kappa V_{\mathrm{g}} / U_{\mathrm{T}}}\right) \frac{d V_{\mathrm{g}}}{d t}, \\
& =\frac{\kappa}{U_{\mathrm{T}}} I_{\text {out }} \frac{d V_{\mathrm{g}}}{d t},
\end{aligned}
$$

which allows us to rewrite (31) as

$$
\begin{gathered}
\frac{C U_{\mathrm{T}}}{\kappa I_{\mathrm{b}}} \frac{d I_{\text {out }}}{d t}=I_{\text {out }}\left(1-\frac{I_{\text {out }}}{I_{\mathrm{b}}}\right), \\
\tau \frac{d I_{\text {out }}}{d t}=I_{\text {out }}\left(1-\frac{I_{\text {out }}}{I_{\mathrm{b}}}\right) .
\end{gathered}
$$

The time constant is identified as $\tau=\left(C U_{\mathrm{T}}\right) /\left(\kappa I_{\mathrm{b}}\right)$.

Equation (35) happens to be the logistic equation, a simple model of population dynamics. It can be solved exactly either by separation of variables followed by partial fractions, or by solving it as Bernoulli's equation. The solution is

$$
I_{\text {out }}(t)=\frac{I_{\mathrm{b}} e^{t / \tau}}{e^{t / \tau}-1+I_{\mathrm{b}} / I_{\mathrm{out}_{0}}},
$$

where $I_{\text {out }}$ is the initial value of $I_{\text {out }}$. We are lucky to have an exact solution to (35), given that it is a nonlinear differential equation. Even so, it is difficult to discern much useful information about $I_{\text {out }}$ 's qualitative behavior from (36). For instance, it is not clear how the behavior of $I_{\text {out }}$ might change with different initial conditions. To answer questions of this sort, it is helpful to do geometric analysis on the system's corresponding vector field.

Since the simple current mirror is a one-dimensional system, its vector field is represented as a flow on a line. The direction and speed of the flow are dictated by the right hand side (RHS) of (35). It is a quadratic, as shown in Figure 10. The $I_{\text {out }}$-intercepts are 0 and $I_{\mathrm{b}}$. There is a maximum at $I_{\text {out }}=I_{\mathrm{b}} / 2$. The vector field is depicted as the arrows on the $I_{\text {out }}$ axis. For positive values of $d I_{\text {out }} / d t, I_{\text {out }}$ is increasing, meaning the arrows point to the right. For negative values of $d I_{\text {out }} / d t, I_{\text {out }}$ is decreasing, meaning the arrows point to the left. When $d I_{\text {out }} / d t=0$, there is no change in $I_{\text {out }}$ and the circuit is said to be at equilibrium.

The vector field provides clear, qualitative information about the behavior of $I_{\text {out }}$. There are two equilibrium points, namely $I_{\text {out }}=0$ and $I_{\text {out }}=I_{\mathrm{b}}$. Note that the vector field flows away from $I_{\text {out }}=0$. This equilibrium point is unstable, since the system will not recover from slight disturbances away from it. The vector field flows towards $I_{\text {out }}=I_{\mathrm{b}}$, implying 


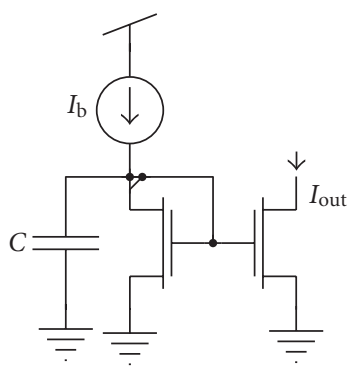

(a)

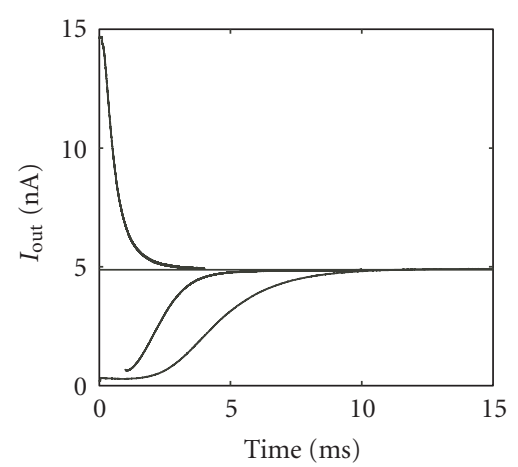

(b)

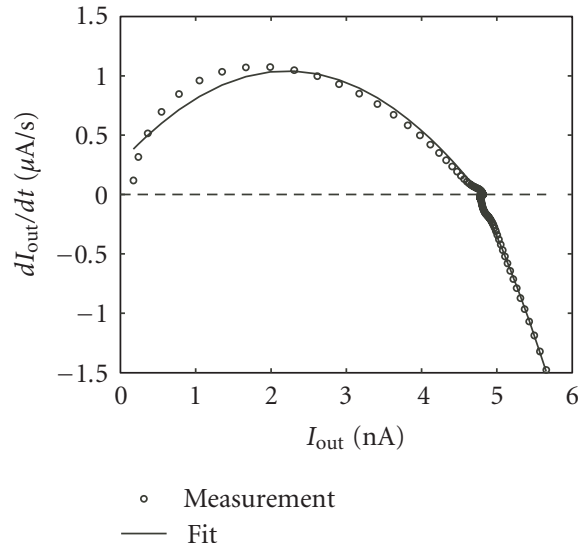

(c)

FIGURE 8: Simple current mirror. (a) Circuit that was compiled onto the FPAA. (b) Measured trajectories for different initial conditions. (c) Vector field derived from trajectory measurements. The origin is an unstable equilibrium point, while 5 is stable.

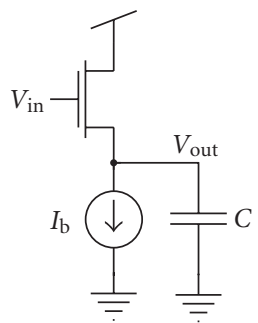

(a)

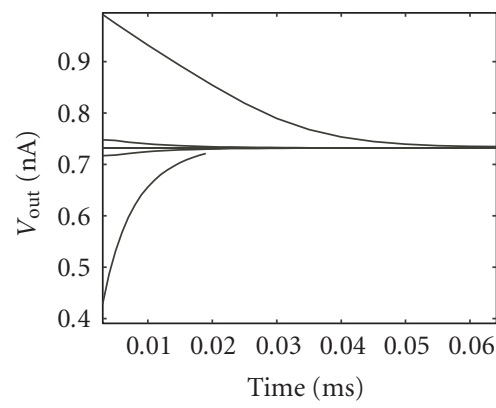

(b)

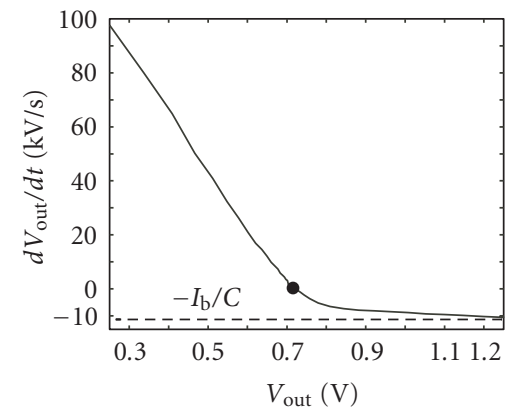

(c)

Figure 9: Source Follower Amplifier Acting as a Simple Peak Detector. (a) Circuit that was compiled onto the FPAA. (b) Measured step responses. (c) Vector field derived from step response measurements. The point $(0,0.7)$ is a stable fixed point.

that this is a stable equilibrium point. If the system is initially at $I_{\text {out }}=I_{\mathrm{b}}$ and then experiences a small disturbance, it will tend back to the $I_{\text {out }}=I_{\mathrm{b}}$ point.

The vector field in Figure 10 also gives information about the acceleration of $I_{\text {out }}$ as it approaches the $I_{\text {out }}=I_{\mathrm{b}}$ equilibrium point. For $0<I_{\text {out }}<I_{\mathrm{b}} / 2$, the rate of change of $I_{\text {out }}$ increases until it reaches a peak at $I_{\text {out }}=I_{\mathrm{b}} / 2$. Between $I_{\mathrm{b}} / 2$ and $I_{\mathrm{b}}$, the system decelerates until the rate of change of $I_{\text {out }}$ eventually becomes zero. For $I_{\text {out }}>I_{\mathrm{b}}$, the rate of change of $I_{\text {out }}$ steadily decreases until $I_{\text {out }}=I_{\mathrm{b}}$. It is interesting to note that, for $I_{\text {out }}<I_{\mathrm{b}}$, the rate of change of $I_{\text {out }}$ is limited to a maximum of $I_{\mathrm{b}} /(4 \tau)$.

The geometric analysis predictions can be checked against experimental measurements of a current mirror that was compiled onto an FPAA. Figure 8(b) depicts various trajectories, or solutions, of the system of (35) for different initial conditions. Notice that trajectories that start at values lower than $I_{\text {out }}=I_{\mathrm{b}} / 2$ have a sigmoidal shape, with the point of inflection corresponding to the maximum rate of change of current $d I_{\text {out }} / d t=I_{\mathrm{b}} /(4 \tau)$. The parabolic shape of $d I_{\text {out }} / d t$ can be extracted from these trajectories, and it is shown in Figure 8(c).
8.2. Simple Peak Detector. Assuming subthreshold operation, the KCL equation for the source follower amplifier of Figure $9(\mathrm{a})$ is the following.

$$
C \frac{d V_{\text {out }}}{d t}=I_{\mathrm{o}} e^{\left(\kappa V_{\text {in }}-V_{\text {out }}\right) / U_{\mathrm{T}}}-I_{\mathrm{b}} .
$$

Note that

$$
\frac{d}{d t} e^{V_{\text {out }} / U_{\mathrm{T}}}=\frac{e^{V_{\text {out }} / U_{\mathrm{T}}}}{U_{\mathrm{T}}} \frac{d V_{\text {out }}}{d t},
$$

in which case, the solution to (37) is

$$
V_{\text {out }}=\kappa V_{\text {in }}+U_{\mathrm{T}} \log \left(\frac{I_{\mathrm{o}}}{I_{\mathrm{b}}}-\left(\frac{I_{\mathrm{o}}}{I_{\mathrm{b}}}-e^{\left(V_{\text {out }_{0}}-\kappa V_{\text {in }}\right) / U_{\mathrm{T}}}\right) e^{-t / \tau}\right),
$$

where $\tau=C U_{\mathrm{T}} / I_{\mathrm{b}}$ and $V_{\text {out }_{0}}$ is the initial value of $V_{\text {out }}$.

The time that it takes for $V_{\text {out }}$ to be within $10 \%$ of its final value is

$$
t_{10}=\tau \log \left|\frac{I_{\mathrm{o}} / I_{\mathrm{b}}-e^{\left(V_{\text {out }_{0}}-\kappa V_{\text {in }}\right) / U_{\mathrm{T}}}}{I_{\mathrm{o}} / I_{\mathrm{b}}-e^{0.1 \kappa V_{\text {in }} / U_{\mathrm{T}}}}\right| .
$$


For a large positive step input, $e^{\left(V_{\text {out }_{0}}-\kappa V_{\text {in }}\right)} \approx 0$, and $(40)$ is approximately

$$
t_{10}=t_{10+} \approx \tau \log \left|\frac{I_{\mathrm{o}}}{I_{\mathrm{o}}-I_{\mathrm{b}} e^{0.1 \kappa V_{\mathrm{in}} / U_{\mathrm{T}}}}\right| .
$$

For a large negative step input, $e^{\left(V_{\text {out }_{0}}-\kappa V_{\text {in }}\right)} \gg I_{\mathrm{o}} / I_{\mathrm{b}}$, and (40) becomes

$$
\begin{aligned}
t_{10}=t_{10-} & \approx \tau \log \left|\frac{I_{\mathrm{b}} e^{\left(V_{\mathrm{out}_{0}}-\kappa V_{\text {in }}\right)}}{I_{\mathrm{o}}-I_{\mathrm{b}} e^{0.1 \kappa V_{\mathrm{in}} / U_{\mathrm{T}}}}\right| \\
& =t_{10+}+\tau\left(\frac{V_{\mathrm{out}_{0}}}{U_{\mathrm{T}}}-\kappa \frac{V_{\text {in }}}{U_{\mathrm{T}}}\right) \log \left(\frac{I_{\mathrm{b}}}{I_{\mathrm{o}}}\right) .
\end{aligned}
$$

Equations (41) and (42) indicate that the response of the peak detector is slower for a negative input step than it is for a positive input step. We surmise that if the input is continuously varying at a rate faster than $1 /\left(t_{10-}\right)$, then the output will be a reasonable representation of the input's peak values. Explaining the peak detector's behavior with (41) and (42) is rigorous, but depends on having to manipulate the expression of (39).

One way of avoiding the math is to employ intuitive descriptions of the charging action of the active device (i.e., the transistor) versus the discharging action of the current source [12]. A more rigorous approach is to apply nonlinear geometric analysis to the problem. Consider the plot of $d V_{\text {out }} / d t$ versus $V_{\text {out }}$ shown in Figure 9 (c). We constructed it from a number of step response measurements (Figure 9(b)) that we took after compiling the source-follower amplifier onto the FPAA. A large negative input step corresponds to an initial value of $V_{\text {out }_{0}} \gg V_{\text {in. }}$. The rate of growth of $V_{\text {out }}$ is bounded by $I_{\mathrm{b}} / C$. For a large positive input step, however, $V_{\text {out }_{0}} \ll V_{\text {in }}$, and the maximum rate at which $V_{\text {out }}$ approaches $V_{\text {in }}$ can be much greater than $I_{\mathrm{b}} / C$. The maximum rate of approach in this case is limited only by the initial value, $V_{\text {out }_{0}}$. As such, there is an asymmetry in the speed of the circuit's response to up-going versus downgoing movements on the input. The effect of this asymmetry is that $V_{\text {out }}$ tracks increasing $V_{\text {in }}$ and not decreasing $V_{\text {in }}$, which is the behavior of a peak detector.

\section{A Two-Dimensional System}

Figure 11 depicts Lyon and Mead's classic second-order section [13]. It is a Gm-C filter with two poles that can be placed anywhere on the real/imaginary plane. We begin our analysis by writing down the governing equations for the circuit, assuming that the OTAs are based on subthreshold MOS transistor differential pairs:

$$
\begin{aligned}
& C_{1} \frac{d V_{2}}{d t}=\frac{I_{2}}{k} \tanh \left(\frac{\kappa\left(V_{1}-V_{2}\right)}{2 U_{\mathrm{T}}}\right), \\
& C_{1} \frac{d V_{1}}{d t}=I_{1} \tanh \left(\frac{\kappa\left(V_{\mathrm{in}}-V_{1}\right)}{2 U_{\mathrm{T}}}\right)-I_{3} \tanh \left(\frac{\kappa\left(V_{2}-V_{1}\right)}{2 U_{\mathrm{T}}}\right),
\end{aligned}
$$

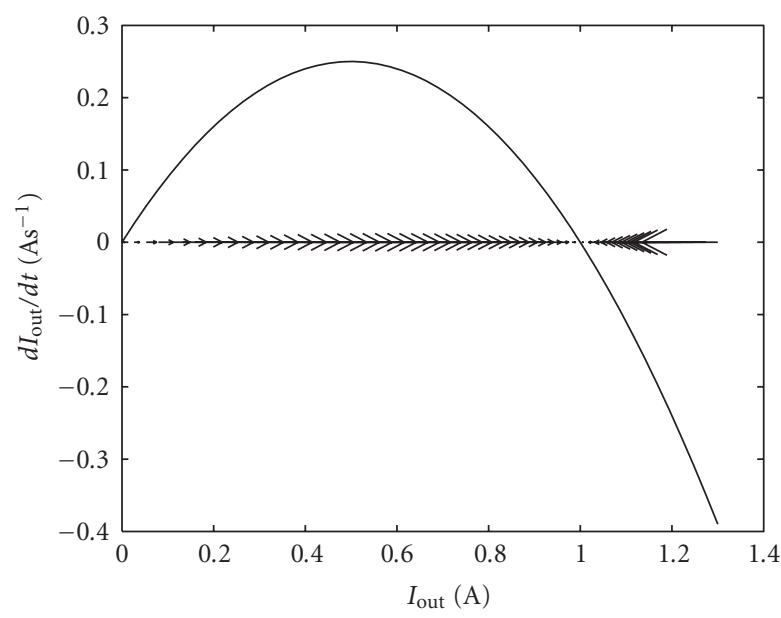

FIGURE 10: The vector field of the logistic equation is represented as a flow on the $I_{\text {out }}$ axis. For positive values of $d I_{\text {out }} / d t, I_{\text {out }}$ is increasing and the flow is to the right. For negative values of $d I_{\text {out }} / d t, I_{\text {out }}$ is decreasing and the flow is to the left.

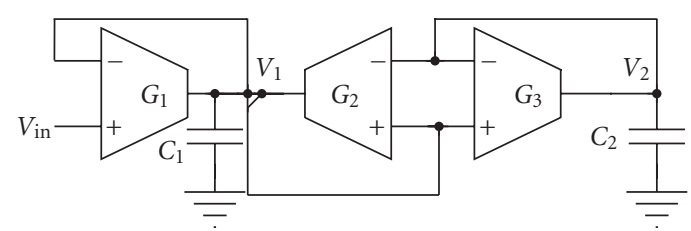

Figure 11: Second-Order Section. Varying the bias currents of the various amplifiers leads to interesting dynamics.

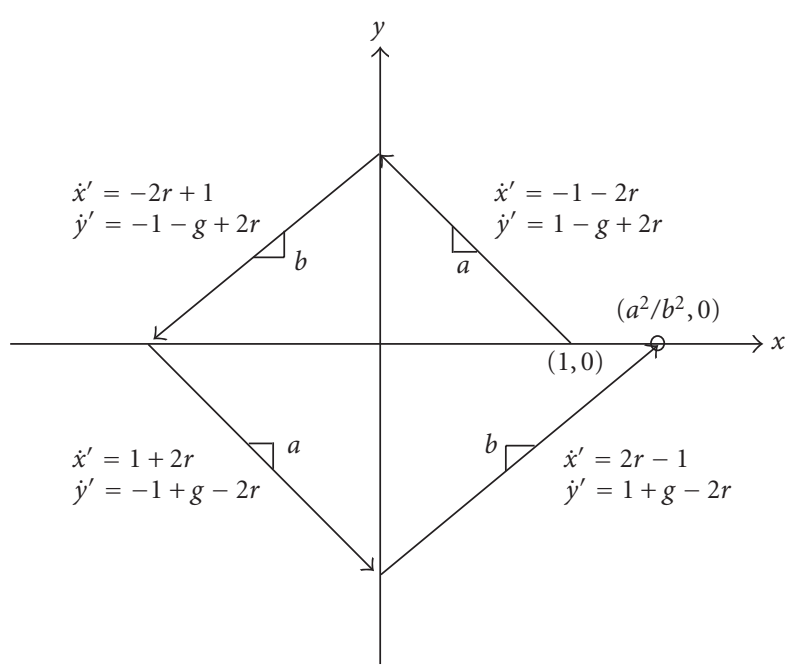

FIGURE 12: Sketch of SOS Phase Plane for Large Signals.

where $I_{1,2,3}$ are the bias currents of the OTAs. Also, $k$ is the ratio of the $C_{2}$ to $C_{1}$.

If we define

$$
x=\frac{\kappa\left(V_{1}-V_{\text {in }}\right)}{2 U_{\mathrm{T}}}, \quad y=\frac{\kappa\left(V_{2}-V_{1}\right)}{2 U_{\mathrm{T}}},
$$




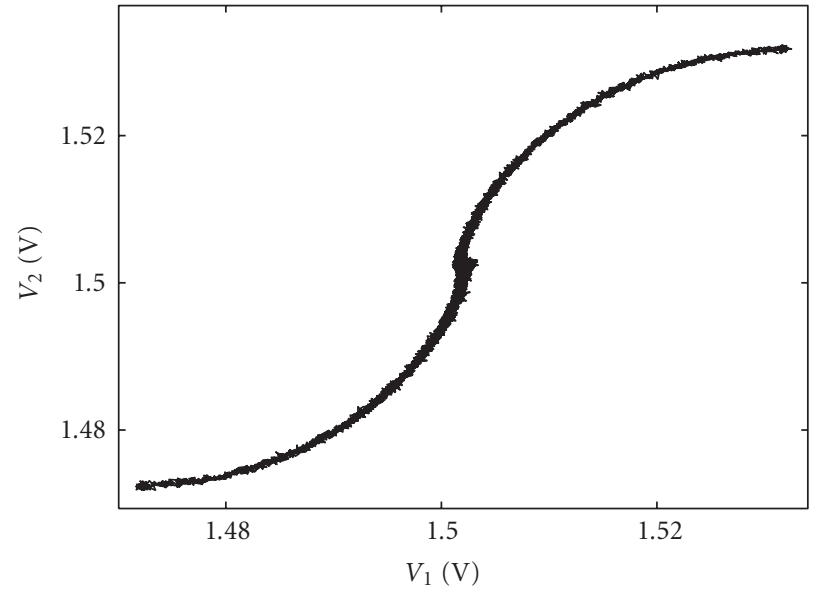

(a)

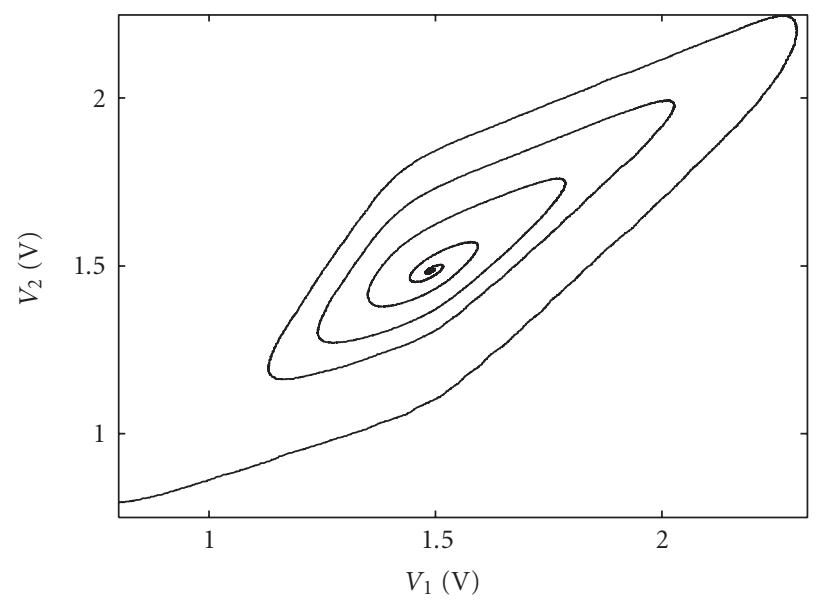

(c)

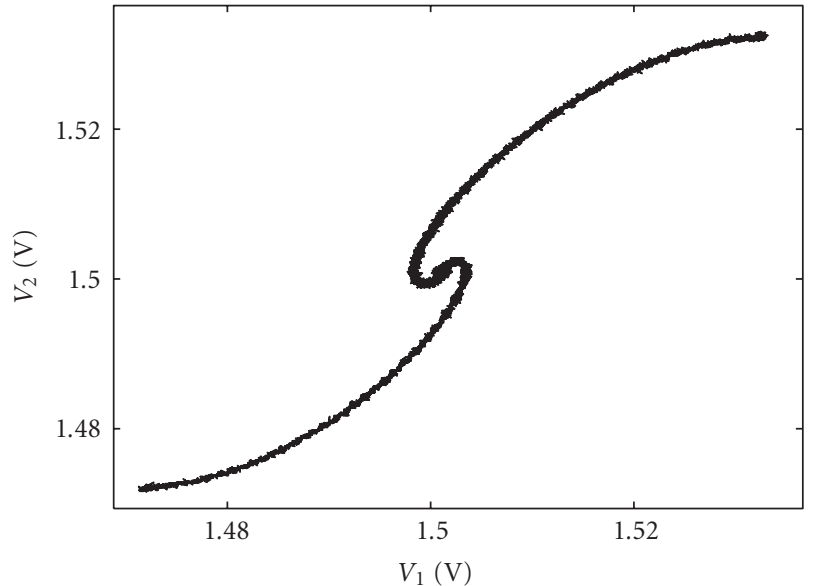

(b)

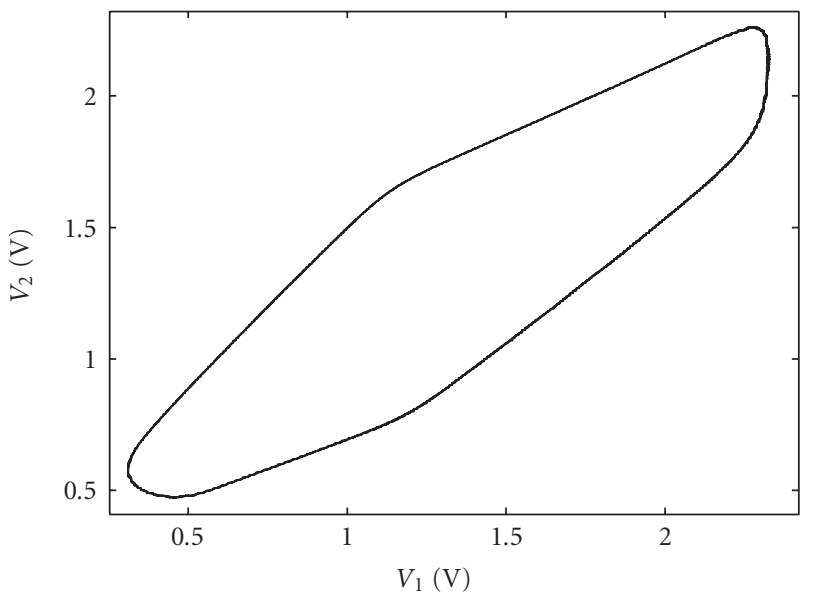

(d)

Figure 13: SOS Experimental Phase Plane Results for Various Values of $r$.

then (43) become

$$
\begin{gathered}
\frac{2 U_{\mathrm{T}} C_{1}}{\kappa} \frac{d x}{d t}=-I_{1} \tanh (x)-I_{3} \tanh (y), \\
\frac{2 U_{\mathrm{T}} C_{1}}{\kappa} \frac{d y}{d t}=I_{1} \tanh (x)+\left(I_{3}-\frac{I_{2}}{k}\right) \tanh (y) .
\end{gathered}
$$

Further defining

$$
\begin{gathered}
I_{1}=I_{\text {bias }}, \\
I_{2}=g k I_{\text {bias }}, \\
I_{3}=2 r I_{\text {bias }}, \\
t=\tau \cdot \frac{2 U_{\mathrm{T}} C_{1}}{\kappa I_{\text {bias }}},
\end{gathered}
$$

where $g \geq 0$, we get the following dimensionless equation

$$
\begin{gathered}
\frac{d x}{d \tau}=-\tanh (x)-2 r \tanh (y), \\
\frac{d y}{d \tau}=\tanh (x)+(2 r-g) \tanh (y) .
\end{gathered}
$$

9.1. Small Signal Analysis. We can linearize (47) by replacing the RHS with its Jacobian, giving

$$
\left[\begin{array}{l}
\dot{x} \\
\dot{y}
\end{array}\right] \approx\left[\begin{array}{cc}
-1 & -2 r \\
1 & 2 r-g
\end{array}\right]\left[\begin{array}{l}
x \\
y
\end{array}\right] .
$$

The origin is a fixed point. In fact, it is a unique fixed point, since (from (47)) $\tanh (x)=-2 r \tanh (y) \Longrightarrow x=y=0$. The origin is stable for

$$
r<\frac{1+g}{2}
$$

and unstable otherwise. It is a spiral for

$$
\frac{1+g}{2}-\sqrt{g}<r<\frac{1+g}{2}+\sqrt{g}
$$

and a node otherwise.

9.2. Large Signal Analysis. For certain values of $r$ and $g$, the nonlinearities of the second-order section causes it to suffer instability. In this region of parameter space, linear 
analysis accurately predicts that the circuit is small signal stable, but completely fails to recognize that instability would occur for large signals. Mead addresses this issue in [14], but we will present a somewhat more thorough treatment of the problem, using phase-plane analysis and experimental verification with the FPAA.

For very large values of $x$ and $y$, the tanh functions get saturated, and can each be approximated with a signum function. Equation (47) becomes

$$
\begin{gathered}
\frac{d x}{d \tau}=-\operatorname{sgn}(x)-2 r \operatorname{sgn}(y), \\
\frac{d y}{d \tau}=\operatorname{sgn}(x)+(2 r-g) \operatorname{sgn}(y) .
\end{gathered}
$$

Figure 12 shows the phase plane ( $x$ versus $y$ ) that corresponds to (51). The depicted motion is valid only if $1 / 2<$ $r<1$. The gradient in the first and third quadrants is

$$
a=\frac{2 r-1+g}{1+2 r}
$$

and that in the second and fourth quadrants is

$$
b=\frac{2 r-1-g}{1-2 r} .
$$

Observe that, with an initial condition of $(1,0),(51)$ predicts that the positive $x$-axis will again be intercepted at $\left(a^{2} / b^{2}, 0\right)$. If $a^{2} / b^{2}>1$, then $x$ and $y$ will grow without bound. Stated in terms of the $r$ and $g$ variables, there is large signal instability if

$$
r>\frac{g+\sqrt{g^{2}+4}}{4} .
$$

Our analysis of the second-order section can readily be verified experimentally. We compiled the filter on the FPAA, as shown in Figure 7. The bias currents of all three OTAs are user-programmable, and varying them corresponds to varying the values of $r$ and $g$. The FPAA thus allows us to explore the parameter space of the filter, and to observe changes in its qualitative behavior. It can effectively be used for bifurcation analysis.

Figure 13 shows the filter's phase plane plots for various values of $r$, with $g$ kept fixed. Just as we predicted, there is a unique fixed point, which is initially stable, and gradually changes from a node to a spiral (Figures 13(a) and 13(c)). While linear analysis would predict these three responses as damped, slightly underdamped, and very underdamped, it fails to recognize the possibility of the fourth response, which is large-signal instability. In the fourth panel, $r$ meets the criterion derived from nonlinear analysis, (54), and we observe oscillation. Further analysis and exploration of parameter space reveals that this second-order section is capable of low-distortion sinusoidal oscillation [15]. Such functionality is valuable in communication systems.

\section{Conclusion}

In this paper, we have introduced visual and graphical techniques for analyzing nonlinear circuit dynamics. Our approach to studying harmonic distortion yields information about the various processing flows that are responsible for each harmonic term. The FPAA was used to rapidly create phase plane plots, which concisely encapsulate the nonlinear dynamics of the circuit under study. We have provided various examples of our techniques and have compared our predictions to experimentally-measured data.

\section{References}

[1] R. Sarpeshkar, M. W. Baker, C. D. Salthouse, J.-J. Sit, L. Turicchia, and S. M. Zhak, "An analog bionic ear processor with zero-crossing detection," in Proceedings of IEEE International Solid-State Circuits Conference (ISSCC '05), vol. 48, pp. 78-79, San Francisco, Calif, USA, February 2005.

[2] F. Serra-Graells, L. Gomez, and J. L. Huertas, "A true-1V 300-W CMOS-subthreshold log-domain hearing-aid-onchip," IEEE Journal of Solid-State Circuits, vol. 39, no. 8, pp. 1271-1281, 2004.

[3] F. Marton and R. Säljö, "On qualitative differences in learning 1: outcome and process," British Journal of Educational Psychology, vol. 46, pp. 4-11, 1976.

[4] R. D. Middlebrook, "Low-entropy expressions: the key to design-oriented analysis," in Proceedings of 21st Frontiers in Education Conference, pp. 399-403, West Lafayette, Ind, USA, September 1991.

[5] T. A. DeFanti, M. D. Brown, and B. H. McCormick, "Visualization: expanding scientific and engineering research opportunities," Computer, vol. 22, no. 8, pp. 12-16, 1989.

[6] K. Odame and P. E. Hasler, "Harmonic distortion analysis via perturbation methods," in Proceedings of the 51st Midwest Symposium on Circuits and Systems, pp. 554-557, Knoxville, Tenn, USA, August 2008.

[7] A. Buonomo and A. L. Schiavo, "Perturbation analysis of nonlinear distortion in analog integrated circuits," IEEE Transactions on Circuits and Systems I, vol. 52, no. 8, pp. 16201631, 2005.

[8] R. Rand, Lecture Notes on Nonlinear Vibrations, The InternetFirst University Press, Ithaca, NY, USA, 2003.

[9] S. H. Strogatz, Nonlinear Dynamics and Chaos, Westview, Cambridge, Mass, USA, 1994.

[10] C. C. Enz, F. Krummenacher, and E. A. Vittoz, "An analytical MOS transistor model valid in all regions of operation and dedicated to low-voltage and low-current applications," Analog Integrated Circuits and Signal Processing, vol. 8, no. 1, pp. 83-114, 1995.

[11] C. M. Twigg and P. Haslet, "A large-scale reconfigurable analog signal processor (RASP) IC," in Proceedings of the IEEE Custom Integrated Circuits Conference (CICC '06), pp. 5-8, September 2006.

[12] B. P. Lathi, Modern Digital and Analog Communication Systems, Oxford University Press, New York, NY, USA, 1998.

[13] R. F. Lyon and C. Mead, "An analog electronic cochlea," IEEE Transactions on Acoustics, Speech, and Signal Processing, vol. 36, no. 7, pp. 1119-1134, 1988.

[14] C. Mead, Analog VLSI and Neural Systems, Addison-Wesley, Reading, Mass, USA, 1989.

[15] K. Odame and P. Hasler, "Exploiting ota nonlinearity in the design of a second order section oscillator," in Proceedings of the RISP International Workshop on Nonlinear Circuits and Signal Processing, pp. 5-8, March 2006. 

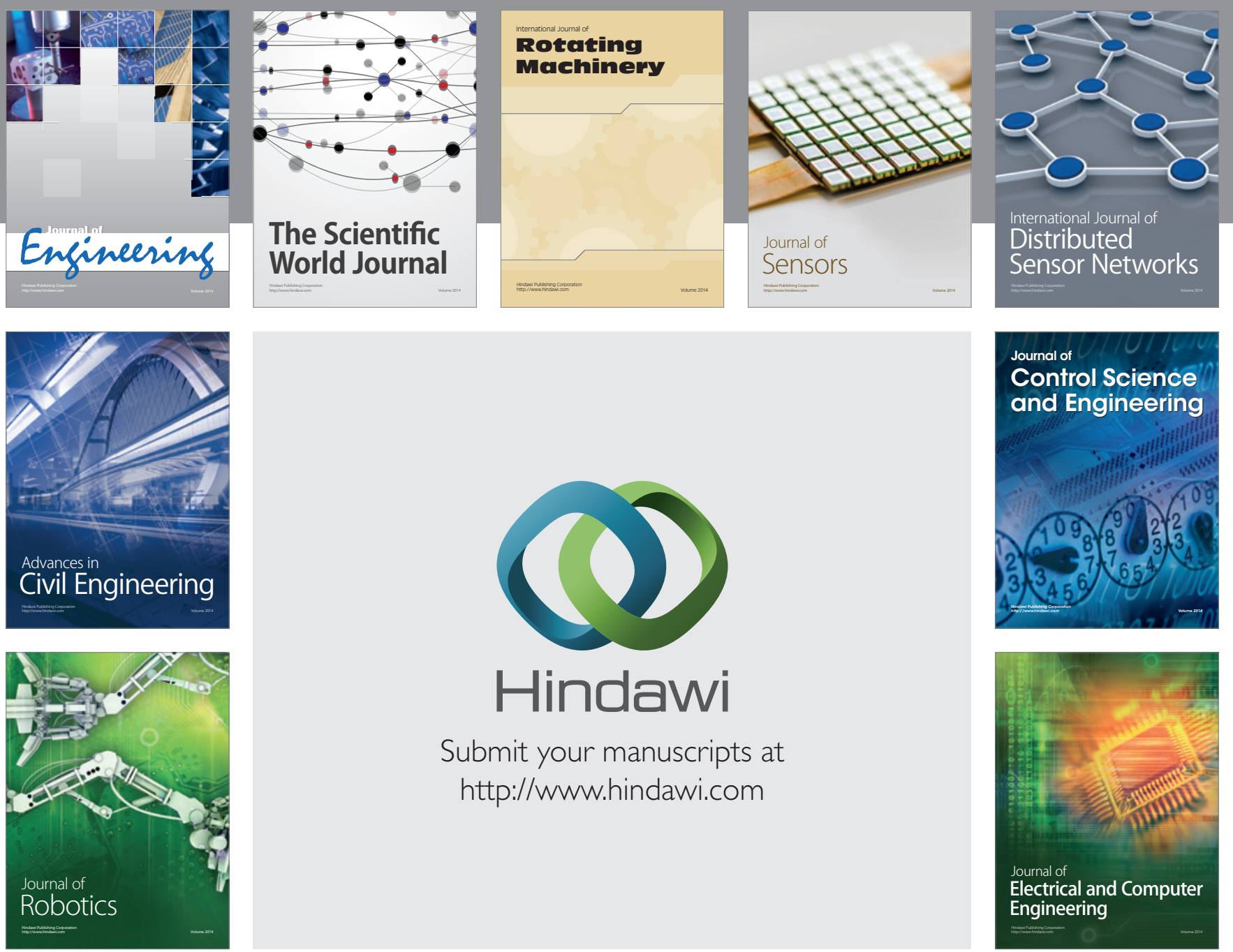

Submit your manuscripts at

http://www.hindawi.com
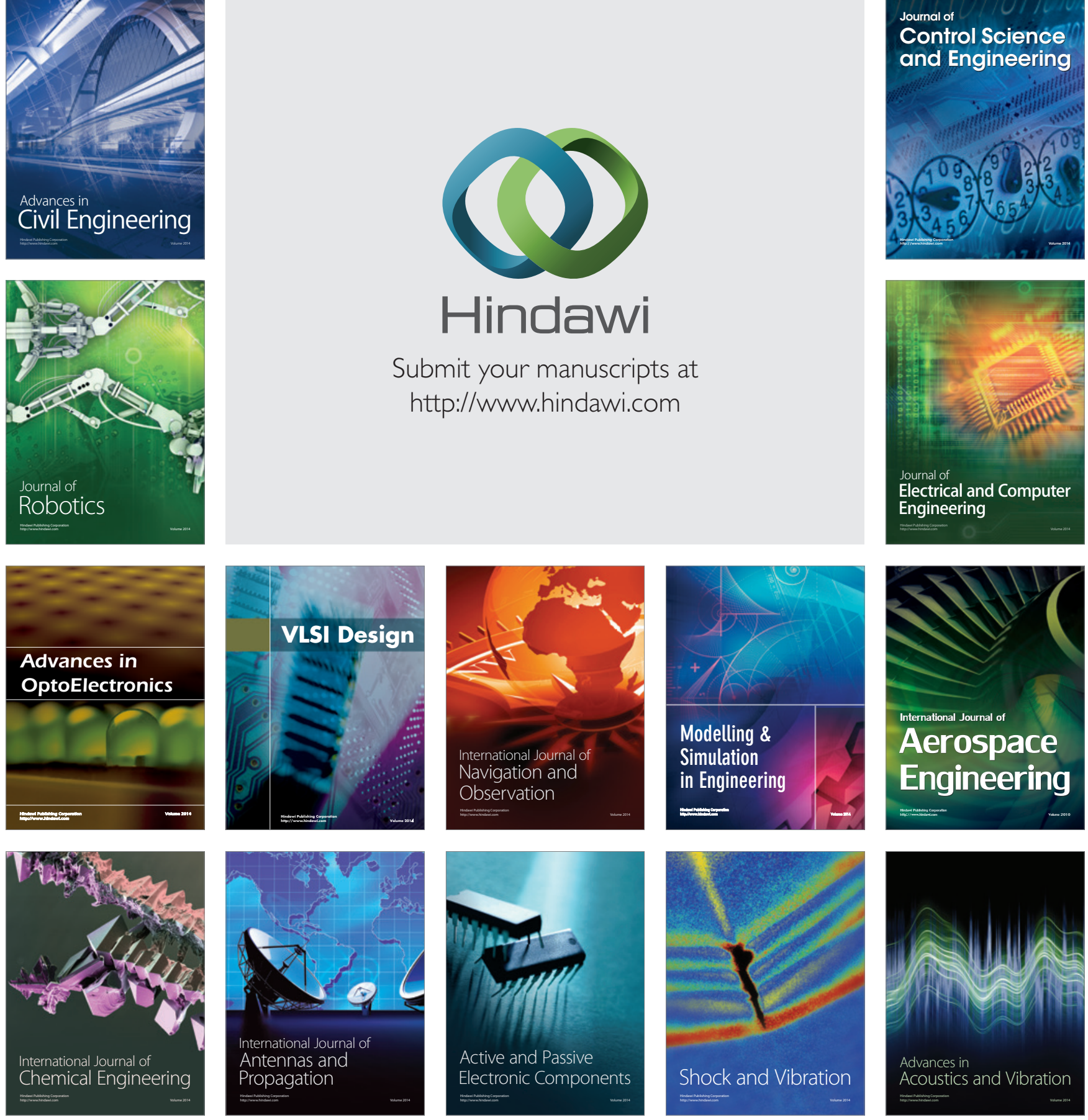